\title{
Carnets
}

Revue électronique d'études françaises de l'APEF

Première Série - 2 Numéro Spécial | 2010

Littératures nationales: suite ou fin. Résistances, mutations \& lignes de fuite

\section{À la recherche de l'identité perdue (essai sur la crise d'identité dans le roman portugais contemporain)}

\section{Maria de Fátima Marinho}

\section{OpenEdition}

Journals

Édition électronique

URL : http://journals.openedition.org/carnets/5313

DOI : 10.4000/carnets.5313

ISSN : 1646-7698

Éditeur

APEF

Édition imprimée

Date de publication : 1 juin 2010

Pagination : 243-253

\section{Référence électronique}

Maria de Fátima Marinho, «À la recherche de l'identité perdue (essai sur la crise d'identité dans le roman portugais contemporain) », Carnets [En ligne], Première Série - 2 Numéro Spécial | 2010, mis en ligne le 16 juin 2018, consulté le 19 avril 2019. URL : http://journals.openedition.org/carnets/5313 ; DOI : 10.4000/carnets.5313

Ce document a été généré automatiquement le 19 avril 2019

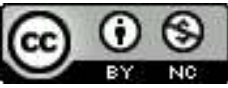

Carnets est mis à disposition selon les termes de la licence Creative Commons - Atribution - Pas d'utilisation commerciale 4.0 International. 


\title{
À la recherche de l'identité perdue (essai sur la crise d'identité dans le roman portugais contemporain)
}

\author{
Maria de Fátima Marinho
}

1 Les interférences qui s'établissent entre les faits, les événements et leur récit (indépendamment de la distance temporelle séparant les uns des autres) sont le signe évident de leur interdépendance, sans compter la quasi inexistence des premiers s'ils ne sont pas fondés sur une espèce de mémoire qui, tout en pouvant assumer différentes nuances, ne cesse jamais de constituer une borne fondamentale pour l'émergence de la notion d'identité, si importante dans la formation de l'idée de nationalité et sa légitimation. La certitude que l'Histoire est née le jour où un homme a raconté sa vie à un autre homme était déjà énoncée par Alfred de Vigny, $1872^{1}$, et s'avère condition nécessaire à l'entendement de la fascination exercée par le souvenir et son rôle central dans le travail d'écriture des grands récits qui, cycliquement, sont protagonistes du devenir littéraire. Aussi nous semble-t-il pertinent de dire que le pouvoir de la narration dans la pratique historiographique est variable et complexe; il est possible de l'aborder selon différents points de vue qui embrassent des disciplines comme la rhétorique, l'histoire et la grammaire ${ }^{3}$. La mémoire constitue ainsi la catégorie nécessaire à travers laquelle la narration écrit l'Histoire, et le travail romanesque en est l'outil qui permet de déplacer le passé vers le plan du présent et qui reconfigure l'identité en danger d'effondrement ${ }^{4}$. C'est dans le cadre de cette reconfiguration que se pose le problème de la vérité, dans la mesure où les faits historiques peuvent être considérés comme de simples interprétations subjectives ${ }^{5}$, construites par des sujets plus ou moins inféodés à des idéologies, des préjugés ou des intérêts. L'éventuelle manipulation de l'événement agit en influençant l'opinion du lecteur, favorisant l'oubli de ce qui pourrait modifier les savoirs conformes ${ }^{6}$. Cette manipulation est, par ailleurs, loin de pouvoir être considérée inoffensive car, de fait, elle finit par conditionner la lecture du passé, suscitant l'éclosion d'idées reçues et d'explications inébranlables. Même si l'écriture est mode de reconstruction du passé, elle est avant tout responsable de la création du concept d'identité nationale, cette notion ne pouvant exister a priori mais s'avérant fondamentale 
pour la légitimation de nombre de comportements et d'attitudes. L'identité est, en effet, un point de convergence d'aspects divers, tels que la tradition, les mythes ou les tentatives de différenciation à l'égard de l'autre, perçu comme un ennemi ou comme un adversaire des volontés communautaires ${ }^{7}$. C'est dans cette perspective que le passé se transforme en un élément d'apaisement des tensions, un élément commode par rapport à un présent complexe et face à un futur inconfortable ${ }^{8}$.

Cette ambiguïté de l'homme par rapport au monde, actualisée dans l'écriture de l'Histoire 9 , permet de comprendre l'émergence du roman historique comme grande narration qui favorise l'esprit national et aide à minimiser les effets négatifs de moments de crise. Ce type de texte surgit au début du XIXe siècle, lorsqu'une grande partie des pays européens se sont sentis menacés par les changements intempestifs issus de la Révolution Française et par les tentatives d'agression des armées napoléoniennes auprès de souverainetés établies depuis longtemps. La conjoncture politique dérivée de ces événements, qui engendra un pouvoir prioritairement exercé par la classe bourgeoise et déclencha un débat, bien qu'implicite, sur la pertinence de l'identité - affaiblie par la constatation de la faillite des modèles politiques mis en échec par les circonstances - a mené à l'apparition d'un récit capable d'agglutiner à la fois des facteurs de légitimation historique et des éléments d'intervention sur un présent criblé d'incertitudes et de frustrations.

La mode inaugurée par Walter Scott se répand un peu partout en Europe et, au Portugal, elle atteint son sommet avec l'œuvre d'Alexandre Herculano qui, à travers les textes réunis sous le titre Lendas e Narrativas ("Légendes et Récits") et trois romans, intitulés Eurico o Presbitero, O Monge de Cister et O Bobo ("Eurico, l'Ecclésiastique", "Le Moine de Cister" et "Le Bouffon"), essaye d'éduquer le peuple, en démontrant l'existence d'une identité propre, construite depuis des siècles et sans doute fondamentale pour l'établissement d'un gouvernement constitutionnel. Le souci didactique, si cher aux romantiques, fruit du transfert du pouvoir politique et économique - ce transfert s'avérant malaisé au niveau du pouvoir intellectuel -, avait pour but d'inculquer l'idée d'une transcendance du passé dans la construction d'un présent semé d'écueils. En l'absence d'une recherche historique - que la population, plus préoccupée par l'enrichissement que par l'étude, ne privilégiait point - le romancier montre les événements passés sous le masque de la fiction. Des déclarations d'Alexandre Herculano et Arnaldo da Gama, entre autres, démontrent clairement les intentions pédagogiques de leurs ouvrages et mettent à nu la fonction de la grande narration qui se donne pour but de remplacer le discours historique proprement dit. L'ingénuité de cette posture ${ }^{10}$, ainsi que l'anachronisme qui en découle, ne sont plus à prouver. La difficulté à transcrire le passé finirait par conduire au refus de cette pratique et à la méfiance à l'égard du récit recelant ces caractéristiques. Au fur et à mesure que le XIXe siècle tend vers sa fin, le roman va altérer substantiellement son rapport à la matière historique. Conscients de ce qu'il y a de factice à placer des personnages romantiques dans des ambiances d'époque réclamant d'autres mentalités et d'autres façons d'agir, les auteurs de la deuxième moitié $\mathrm{du}$ XIXe siècle ont progressivement abandonné le modèle du roman historique traditionnel, pour se concentrer sur l'analyse du présent ou sur la sublimation de facteurs traumatiques. Au Portugal, l'Ultimatum anglais qui, en 1890, exige le renoncement aux prétentions africaines que le Portugal revendiquait, accentue la précarité des gouvernements qui s'avéraient incapables de maintenir la domination outremer, tenue alors pour essentielle. L'activité des mouvements républicains a culminé avec la chute de la monarchie, en 1910, et la succession de gouvernements faibles et faiblement 
représentatifs a favorisé l'éclosion d'un type de grands récits qui visaient, à une époque où les avant- gardes réclamaient déjà leur place au soleil, à consolider l'identité perdue, ou plutôt, l'identité menacée par des circonstances qui portaient atteinte à la fierté nationale. Nous sommes alors en pleine période des romans de deux mille pages, d'auteurs comme Campos Júnior, Rocha Martins, Henrique Lopes de Mendonça ou Artur Lobo d'Ávila, ouvrages romanesques plus soucieux de contrarier la tendance défaitiste que d'entreprendre des expériences sur le plan du langage ou de la technique de narration. Il s'agit de romans laudateurs, excessivement descriptifs, naïvement partiaux, idéologiquement dirigistes, sans doute rigoureux du point de vue factuel mais dépourvus d'intérêt littéraire ou narratif. N'oublions pas que c'est à cette époque que l'avant-garde éclate un peu partout en Europe, refusant entre autres - et ce n'est pas le lieu pour en discuter - la description détaillée et fidèle ${ }^{11}$, l'Histoire (rappelons à ce titre le Manifeste Technique de la Littérature Futuriste, de Marinetti), le récit bien raconté et structuré, homologie idéale d'un monde sans ruptures. C'est peut-être la perception de l'inévitabilité de la rupture - correspondant à la découverte de l'inconscient et de sa nature parcellaire - qui fait que la littérature d'avant-garde privilégie le fragment, le chaos et abandonne, encore que temporairement, la grande narration.

4 Nous ne nous proposons pas d'entreprendre l'analyse du roman au long du XXe siècle ici, même si nous réalisons que, d'une façon ou d'une autre, soit à travers la recherche de l'inconscient, soit par la réflexion existentialiste, soit encore sous le signe de la destruction volontairement assumée par le nouveau roman, il est toujours question de quête d'identité, problématique fuyante et hasardeuse. Nous sommes presque tentés de souscrire aux propos d'Emmanuel Bouju quand il affirme qu'il n'y a pas de différence entre comprendre la réalité et la littérature et que déchiffrer un texte équivaut à élucider le réel ${ }^{12}$, c'est-à-dire que la narration doit accompagner notre façon d'appréhender la réalité, exprimant, directement et indirectement cette recherche.

5 Après plusieurs crises du sens et de ce qu'il signifie conceptuellement, le sujet éprouve le besoin de consolider son expérience destructrice grâce à la présomption d'autres mondes possibles, à la fois cohérents et chaotiques. La post-modernité semble répondre à cette double exigence, en relançant l'histoire bien racontée, sans toutefois escamoter la destruction qu'elle recèle, dans sa genèse même ${ }^{13}$. La double optique inhérente à cette position instaure la voix narratrice autoritaire, puisque productrice de sens, d'un sens qui entretient avec le réel un rapport de contrefactualité ${ }^{14}$.

$6 \mathrm{Au}$ cours des dernières trente années, le roman portugais a privilégié la production de grands récits qui mettent l'accent sur la revisitation du passé, revisitation qui instaure des lectures dynamiques, instigatrices d'une problématisation des connaissances établies mais incessamment interrogées et revitalisées. La transcription de l'Histoire ne saurait être inoffensive, dans la mesure où son approche est d'emblée une forme de responsabilité - dès lors qu'elle interagit avec d'autres modalités littéraires ${ }^{16}$, en un mouvement de légitimation mutuelle et en instaurant un processus de réengagement de l'écriture à travers lequel l'auteur s'expose au jugement extérieur ${ }^{18}$.

7 Ces modalités littéraires finissent par fonctionner comme autant de formes de questionnement du passé, à travers un type de texte que nous pouvons définir comme grand récit, mais qui ne peut plus correspondre au moule des productions du XIXe siècle, puisque la fiabilité de l'écriture a cessé d'être une prémisse valable. Le récit actuel est une sorte de miroir brisé, qui cherche, d'une manière angoissée cependant persistante, l'identité du je et de l'écriture elle-même. Nous nous rangeons du côté d'Emmanuel 
Bouju, lorsqu'il définit les paradoxes du roman actuel: "C'est de cette façon seulement que j'ai voulu appréhender certains des paradoxes auxquels nous sommes confrontés: celui d'un rapport au passé qui privilégie le détour, la référentialisation indirecte, la superposition des époques; celui d'un roman qui au tournant $\mathrm{du} \mathrm{XXI}{ }^{\mathrm{e}}$ siècle demeure familier des modèles d'hier, sans cesse appelés à être remotivés; celui d'une littérature qui cherche volontairement dans son propre répertoire les moyens d'affronter les défis du dicible suscités par l'histoire. ${ }^{\text {"19 }}$

Si nous observons le panorama portugais, il nous sera aisé de trouver des exemples pouvant illustrer les catégories définies par Bouju. Nous nous servirons de quelques romans qui seront prétexte à examiner la manière dont le grand récit se transfigure, au point de réinventer le passé ${ }^{20}$, en tentant d'explorer les interstices inconnus, mais possibles, comme dirait Carlos Fuentes. Dans cette réinvention du passé, nous privilégierons les textes qui se livrent à une quête de l'identité perdue, ceux qui la convoquent constamment, de façons diverses, voire même apparemment déroutantes. Cette quête passe par la réélaboration de figures structurantes de l'imaginaire national, telles Inês de Castro et le roi Sebastião ${ }^{21}$, qui émergent mis en contexte, sous un éclairage critique, dépourvus d'une bonne part de l'aura naïve qu'ils ont acquise au fil des temps.

Pour ce bref essai, nous avons toutefois choisi des textes qui semblent poser de manière plus complexe le problème de l'identité et son rapport à la grande narration, certes fracturée mais nonobstant porteuse de l'espoir que les fragments pourront être rassemblés. Au Portugal, la recherche de l'identité passe par la réconciliation avec le passé colonial, désormais dégagé de la légitimation que la présence impérialiste et dominatrice lui octroyait - ce type de légitimation caractérisant les romans du début du XXe siècle -, et mettant à nu les traumas causés par une retraite peu glorieuse, motif de déceptions profondes et du besoin de les conjurer. Dans ce chapitre précis, nous avons retenu deux romans d'António Lobo Antunes, As Naus ("Les Nefs", 1988) et O Esplendor de Portugal ("La Splendeur du Portugal", 1997), et un ouvrage romanesque de Lídia Jorge, $O$ Vento Assobiando nas Gruas ("Le Vent Sifflant dans les Grues", 2002).

Dans As Naus, Lobo Antunes explore le problème des "retornados" (l'équivalent portugais des pieds noirs) après l'indépendance des anciennes colonies, en leur attribuant les noms des héros des Grandes Découvertes, âge d'or de l'Histoire portugaise. Si Camões, Afonso de Albuquerque, Diogo Cão ou Francisco Xavier sont à présent des misérables à Lisbonne, dénués de toute héroïcité, cela signifie la déchéance d'une identité construite durant des siècles de répétition des mêmes motifs: "Cependant, je ne suis jamais tombé sur des hommes aussi amers qu'à cette époque de douleur où les paquebots rentraient au royaume (reyno) remplis de gens enragés et déçus, un petit paquet à la main pour tout bagage et une aigreur sans cure à la poitrine, humiliés par les anciens esclaves et par la prépotence emplumée des anthropophages. “22.

11 La graphie archaïque du mot portugais "reyno" (royaume) accentue la disparité des époques, qui se confondent en un vertigineux discours, d'où ressortent des caractéristiques peu flatteuses des personnages que nous avens pris l'habitude de vénérer et où se déconstruit l'imaginaire qui apparaît ridiculisé, actualisé en des figures historiques que nous savons porteuses de significations littérales et symboliques.

"Le dénommé Luís en était déjà au tiers de l'écriture de son poème, par un aprèsmidi de septembre où le captieux myope, après de prudents cercles de vautour, le prit par la manche du pyjama et l'invita à assister, la première semaine d'octobre à Ericeir $^{24}$, au débarquement du roi: 
- Sebastião surgit des vagues sur un cheval blanc, siffla-t-il en déposant une rose sur son flacon.

Le poète imagina une horde de phtisiques en uniforme d'hôpital, accroupis sur la brouillasse des dunes, dans l'attente d'un monarque invisible qui monterait des eaux en compagnie de son armée vaincue. ${ }^{\text {"25 }}$

La caricature évidente qui se dégage de ces lignes ne doit susciter une lecture ironique qu'en apparence. L'ironie et la parodie, incontournables, camouflent l'importance de la figuration symbolique, qui semble vouloir détruire, tout en les préservant, les restes éclatés d'une identité qui a du mal à reconnaître son image dans le miroir. Comme chez un personnage d'O Esplendor de Portugal ("La Splendeur du Portugal"), le refus d'accepter le passage du temps correspond, avant tout, à l'incompréhension de l'intégrité du je et, par conséquent, de la Patrie, qui se fissure au fur et à mesure que s'effondrent les fondations fragiles sur lesquelles elle fut bâtie: "Lorsque, le soir, je m'assieds devant la psyché pour enlever le maquillage, je me demande si c'est moi qui ai vieilli ou si c'est plutôt le miroir de la chambre. Ce doit être le miroir: ces yeux ont cessé de m'appartenir, ce visage n'est pas le mien, ces rides et ces taches sur la peau sont-ce des marques de l'âge ou est-ce l'acide d'étain qui corrode la glace?"26

L'hymne national, mis en exergue dans ce roman, n'a évidemment point pour fonction sous-jacente, par exemple, celle qu'il avait dans tous les ouvrages du début du XXe siècle. Il a pour but, ici, de signifier le contraire de ce qu'il énonce, servant ainsi de caution à la thèse que l'on entend démontrer. Dans plusieurs chapitres, les voix narratives se succèdent, en alternance, au sein d'une famille: la mère, vivant encore en Angola, les enfants, déjà à Lisbonne. Les souvenirs obsédants, bourrés de frustrations, de sens occultes, révèlent la nature factice des rapports familiaux, imprégnés de conventions et travaillés par des haines mal déguisées, ainsi que la difficulté à assumer une identité pleine, individuelle ou nationale. L'étrangeté que Carlos - l'ainé, bâtard du père, toléré par la mère, coincé dans un entre deux difficile à gérer, angoissé par l'ambiguïté de la non appartenance à un seul monde (celui des noirs ou celui des blancs) - ressent à l'égard de son propre nom, peut dénoncer cette autre étrangeté qu'est la légitimation de la présence portugaise en Afrique, une légitimation pourtant nécessaire afin que ne se produise point la dispersion complète des fragments d'une identité en danger de dissolution: "Le véritable cœur de la maison étaient les herbes sur les pierres tombales, au soir tombant ou à la nuit naissante, qui disaient des mots que je comprenais mal par peur de comprendre, ce n'était ni le vent, ni les feuilles, mais des voix qui racontaient une histoire traversée par des gens et des bêtes et des meurtres et la guerre, comme si elles chuchotaient incessamment notre faute, nous accusant, répétant des mensonges, comme quoi ma famille et la famille avant la mienne étaient arrivés tels des forbans d'Afrique $($....)"27.

14 Le doute exprimé par la mère au paragraphe précédant a, encore qu'indirectement, pour conséquence les difficultés que Carlos, le métis, éprouve vis à vis de lui-même, l'acculant à passer tout le récit en quête d'une place, une place à chaque fois refusée et à chaque fois accordée:

“(...) me surprenait avec mon nom, disait mon nom

Carlos

Et j'étais autre que ce nom, ce n'était pas mon nom, je ne pouvais pas être ce nom, en

appelant

Carlos

les personnes appelaient un autre Carlos qui était moi en elles et pas moi ni moi en 
moi, c'était un autre doté de la même forme et, s'il leur répondait, ce n'était pas moi qui répondait, c'était leur moi qui parlait, le je en moi se taisait en moi, si bien qu'elles ne connaissaient que leur Carlos à elles, elles ne savaient rien de moi et je restais un étranger, un qui était deux, le leur et le mien, et le mien, étant mien, n'était donc pas, alors il disait comme ils disaient

Carlos

et le leur Carlos à eux n'existait pas pour moi, je me suis souvenu qu'à Luanda ou à la campagne, quand j'écoutais le noir et le silence du noir peuplé de la souffrance des tournesols, c'étaient les seules occasions où, de fait, je dormais avec le je en moi, où je dormais avec moi en répétant

Carlos Carlos Carlos"28

15 La conscience de l'inévitabilité du masque (de la scène), dont nous parlions auparavant ("acteurs déguisés en cadavres, loques déguisées en enfants, jets de polystyrène déguisés

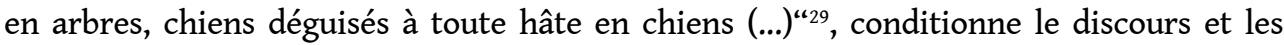
agissements des personnages qui se dépêchent de blanchir leur passé ("les troupes $d u$ Gouvernement et les étrangers de l'Unita n'ont jamais été ici, les bailundos ne se sont jamais enfuis vers la brousse, je n'ai jamais laissé mes enfants sur le quai pour Lisbonne, pas un seul cadavre dans les rues de Luanda, $\left.(. .)^{30}\right)$, même s'ils le font en pleine connaissance de l'imposture qu'ils se proposent d'instaurer.

C'est ce que Lídia Jorge développe magistralement dans O Vento Assobiando nas Gruas ("Le Vent Sifflant dans les Grues"), lorsqu'elle représente une famille d'immigrés du Cap Vert, employant un discours double, à la fois caricatural et vrai. Les rapports que cette famille entretient avec une jeune fille - issue de la haute bourgeoisie de l'Algarve, mais en quelque sorte reniée par les siens, à cause de certains traits de son caractère, tenus pour déviants par rapport à la norme - pose le problème non encore résolu des échanges entre colonisateurs et colonisés. Quand une liaison surgit entre la portugaise, Milène, et un capverdien, la famille accepte tacitement de provoquer l'infertilité forcée de la jeune fille, afin qu'il n'y ait pas d'enfants métis. L'incapacité initiale de Milène à assumer toute seule son statut d'adulte, traduite par la difficulté à construire un discours propre, impliquera l'évolution de son comportement et forcera la conquête d'une identité: "Si elle commençait à appeler - Écoutez, il y a quelqu'un? - cela aurait été le signe qu'elle avait renoncé à trouver, par ses propres moyens, les mots nécessaires à expliquer ce qui s'était passé à Mamie Regina, durant la nuit du quatorze au quinze août, ou comme si elle avait nécessairement besoin des mots des autres pour pouvoir construire sa propre version des faits. "${ }^{\text {"31 }}$

17 L'oscillation entre les mots des uns et les mots des autres, ainsi que l'affirmation de la fausseté du discours ("On a également prétendu que, grâce à l'offre de la clio, la famille Mata, l'ayant trouvée évanouie sur les hortensias propres du microclimat, l'avait sauvée de l'inanition, ce qui ne correspond pas à la vérité. ${ }^{\left({ }^{32}\right)}$ contribuent à engendrer un état d'insécurité des sujets qui s'entêtent à chercher la stabilité d'une identité capable de les réconcilier avec leur passé: "La mort, ce n'est pas mourir, la mort, c'est sortir de la mémoire" ${ }^{33}$.

18 La réconciliation avec le passé passe par l'interpénétration de l'un dans l'autre, en une tentative d'inverser la succession chronologique, disjonctive et opposée aux explications plus complexes que celles issues de la simple causalité. Agustina Bessa-Luís est experte en ce qui concerne la construction de grandes narrations où le présent ne peut être interprété qu'à travers ses liens directs avec le passé et où les personnages ne parviennent à se compléter que par la rencontre de leurs doubles, qui actualisent leurs 
problèmes identitaires. Des ouvrages romanesques comme 0 Mosteiro ("Le Monastère", 1980), o Concerto dos Flamengos ("Le Concert des Flamands") ou Ordens Menores ("Ordres Mineurs”, 1992) constituent autant d'exemples d'explication réciproque, qui passent par la réflexion sur le devenir historique lui-même et l'impossibilité de sa représentation. Comme Nuno Júdice le réfère, dans Por Todos os Séculos ("à Travers les Siècles", 1999), l'évocation du passé est souvent fondée sur des stéréotypes conventionnels, qui apaisent l'instabilité provoquée par l'ignorance effective: "La représentation de Rome était soumise à une vision banale de l'Antiquité, avec les temples, les colonnes simplifiées, l'empereur et les citoyens enveloppés dans des toges" ${ }^{34}$.

Dans son dernier roman, A Ronda da Noite ("La Ronde de Nuit", 2006), Agustina Bessa-Luís croise l'histoire d'une famille avec le célèbre tableau de Rembrandt, tissant des considérations sur ce qu'elle considère la fin d'une ère, au cours de laquelle la place de chacun était prédéterminée, cette place devenant par cela même un facteur d'apaisement et un garant de stabilité: “(...) relique d'un temps révolu, un temps où les privilèges avaient leur mode, $(. . .)^{)^{435}}$.

L'insistance, tout au long du roman, sur l'authenticité ou la fausseté du tableau peut se transformer en un autre doute plus pesant et, du reste, plus dérangeant: l'authenticité d'un peuple qui se bat contre la perte d'identité, sachant que "La liquidation de l'Histoire, un des principaux conflits ayant éclaté lors de la révolution, instigué par des conservateurs qui tenaient à maintenir le peuple éloigné de la réalité" ${ }^{6}$. Des propos comme "Peut-être le Portugal serait-il plus heureux, pauvre et démantelé et inapte à réveiller des idées d'invasion, ou facilement capable d'être pris pour un peuple de bergers. Rien à ajouter au relevé des grandes conquêtes, ni dans l'ordre de l'espace, ni dans l'ordre de la culture. Il avait une histoire noble, mais inconnue. Héros, amants, pensées intraduisibles par l'amour et par l'art" ${ }^{37}$, trouve une sorte d'écho au niveau des doutes quant au (à la) possible meurtrier(ère) de la mère de Juliette, personnage dont l'importance dans la diégèse se doit surtout au fait qu'on la soupçonne d'être matricide (un peu à l'instar du personnage de Silvina - prétendument parricide - du roman Eugénia e Silvina ("Eugénia et Silvina", 1989). La mort d'un des deux êtres, qui atteste notre identité (et l'arrestation de l'autre, libéré seulement nombre d'années après, cette circonstance amenant sa fille à abandonner son mari pour se consacrer entièrement à son père), est symboliquement expliquée par l'absence d'une réelle filiation du tableau - le tableau hante les personnages et en vient à être détruit par quelqu'un qui, littéralement, n'entre pas dans l'histoire. La destruction involontaire de la dite peinture, supposément commise par une employée, scrupuleuse mais ignorante, peut être lue comme la légitimation d'une identité douteuse, dont le roman reflète l'effondrement, à travers des bribes et des débris, porteurs d'images fragmentaires, déformées et trompeuses. C'est le "sens occulte"38, dont parle la narratrice et que le discours tente, apparemment, de dévoiler.

21 Au terme de ce parcours, nous pouvons nous demander où réside et en quoi consiste la pertinence des grandes narrations, miroirs brisés d'un monde chaotique, instable, morcelé. Les exemples que j'ai cités de la production romanesque portugaise contemporaine - et ces exemples auraient pu être multipliés - démontrent le besoin d'atteindre, à travers l'écriture, l'insondable secret du je et de l'autre, qui s'avère fondamentale pour l'établissement d'une identité sous menace constante. Les deux dernières lignes d'A Ronda da Noite nous suggèrent la réponse possible: 
“- Alors vous n'y arrivez pas? Vous n'y arrivez pas?

Il était persuadé de pouvoir y arriver. Comme tout le monde, d'ailleurs." ${ }^{39}$

\section{NOTES}

1. Cf. Alfred de Vigny, "Réflexions sur la Vérité dans l'Art", in Cinq-Mars, Paris, Le Livre de Poche, 1970, (1 ère éd., 1827), p.24: "Le jour où l'homme a raconté sa vie à l'Homme, l'Histoire est née."

2. Cf. Manuel Cruz, "Narrare di Memoria", trad. de Floriana Malacrino, in Gianmario Guidarelli etCarmelo G. Malacrino (organisation), Storia e narrazione - Retorica, Memoria, Immagini, Milan, Bruno Mondadori, 2005, p.70: “(...) si potrebbe contraddistinguere per il fascino verso la memoria o, ancor meglio, verso l'atto in sé del ricordare."

3. Cf., Gianmaria Guidarelli e Carmelo G. Malacrino, "Introduzione", in Gianmario Guidarelli et Carmelo G. Malacrino (organisation), Storia e narrazione - Retorica, Memoria, Immagini, Milan, Bruno Mondadori, 2005, p. 5: "Memoria e oblio, retorica e falsificazione, argomentazione e censura, svelamento e copertura: il ruolo rivestito dalla narrazione nella pratica storiografica è tanto complesso e variegato da poter essere affrontato da tutti questi punti di vista, senza d'altronde riuscire a esaurirne il senso e la portata."

4. Cf., Emmanuel Bouju, La Transcription de l'Histoire - Essai sur le Roman Européen de la Fin du XXe Siècle, Rennes, Presses Universitaires de Rennes, 2006, p.64: "Le retour à la perspective du temps présent depuis l'arrière-plan de l'histoire s'effectue à travers le prisme de la mémoire: dans cette archéologie intérieure, elle est la catégorie subjective nécessaire par laquelle le récit écrit l'histoire, même s'il lui faut pour cela emprunter à autrui ses souvenirs, ou simplement souffrir de leur absence. Le mouvement du roman est celui du retour au passé, ou plus précisément de la réversion du passé en présent: le récit mime le travail archéologique de la mémoire, la découverte des fondations du présent, la reconsidération de soi, et contraint le lecteur effectuer un mouvement analogue dans l'espace du texte. Remémoration construite et reconnaissance permettent à l'écriture de déplacer le temps de l'histoire au plan du présent de lecture: tout se passe comme si l'anamnèse était consciemment conduite, neutralisée et extériorisée, pour être communiquée et éprouvée par le lecteur, jusqu'à l'impératif d'une relecture, d'un retour dans le temps romanesque lui-même."

5. Cf. Hayden White, Tropics of Discourse - Essays in Cultural Criticism, Baltimore e Lenders, The John Hopkins University Press, 1978, p.55: “(...) historical accounts are nothing but interpretations (...)”. 6. Cf., Yves Herat, "Madly, la rhetorical e la "manière d'écrire l'histoire", trad. de Gianmario Guidarelli, in Storia e narrazione - Retorica, Memoria, Immagini, p. 11: "Michel de Certeau defini la storia comme (...) dotata (...) di agire sulle opinioni del lettore, di fargli dimenticare ciò che il racconto lascia da parte (...)".

7. Cf., Giacomo Marramao (entretien avec Manuel Orazi), "Narrazione e Contingenza", in Storia e narrazione - Retorica, Memoria, Immagini, p.21: "La nostra identità può anche esserete immaginata come una sorta di cavea teatrale nella quale riecheggiano le voci delle diverse tradizioni e anche deglin incontri avuti, un sé multiplo e sincronico come nuovo punto di converhenza filosófica, peraltro non nuovo in letteratura."

8. Cf., Manuel da Cruz, art.cit.,p. 73: “(...) il presente risulta svuotato di contenuto, dequalificato a una semplice condizione di osservatore, di contemplatore del passato. Dequalificazione che risulta, in un certo senso, cómoda: (...) Il futuro, viceversa, è profondamente scomodo.". 
9. Cf., Gisèle Séginger, "Introduction", in Ecriture(s) de l'histoire, textes réunis par Gisèle Séginger, Strasbourg, Presses Universitaires de Strasbourg, 2005, p. 11: "Dans les écritures de l'histoire se manifestent les ambiguïtés de la relation de l'homme au monde."

10. Cf. Maria de Fátima Marinho, O Romance Histórico em Portugal, Porto, Campo das Letras, 1999.

11. Cf. André Breton, Manifestes du Surréalisme, Paris, Idées/Gallimard, 1965 [1924], p.16: "Et les descriptions! Rien n'est comparable au néant de celles-ci; ce n'est que superpositions d'images de catalogue (...)".

12. Cf., Emmanuel Bouju, op.cit., p.93: "Il n'y a pas de différence de nature entre comprendre la réalité et comprendre la littérature; déchiffrer le texte enfoui, c'est élucider la violence du réel."

13. Cf. Entretien avec Jasenka Gudelj, "Raccontare la Storia. La Sfida Narrativa di Luther Blisset/ Wu Ming di Luther Blisset/Wu Ming 2", in Storia e narrazione - Retorica, Memoria, Immagini, p. 25: "Noi sentivamo il bisogno di rilanciare una narrative fatta invece di grandi storie, una letteratura "massimalista", in cui ci fossero miti, avventura, leggenda, storia che si intrecciavano tra di loro."

14. Cf. Emmanuel Bouju, op.cit., p.64: "les romans européens de la fin du XX $\mathrm{X}^{\mathrm{e}}$ siècle sont souvent des figures textuelles de l'exemplarité et de ses failles, où la promotion de la voix narrative comme autorité s'accompagne d'une mise en suspens de ses garanties externes."

15. Cf., idem, p.109: “(...) lorsqu'il [le roman] s'évertue à la transcription de l'histoire, se définit lui-même comme une forme sensible de la responsabilité."

16. $\mathrm{Cf}$., idem, ib.: "elle [l'énonciation romanesque] oblige à considérer la visée de l'histoire collective dans son intégration profonde aux modalités de l'échange littéraire."

17. Cf., idem, p.183: "le roman (...) manifeste (...) une nette tendance à risquer sa légitimité dans la reconfiguration esthétique de l'histoire."

18. Cf., idem, p.185: "Et de façon plus générale, l'ambition d'une transcription fictionnelle de l'histoire entraîne souvent un processus de ré-engagement de l'écriture, sur le mode d'une figuration textuelle du geste par lequel l'écrivain s'expose au jugement du monde. L'imitation ostensible d'une scène historiographique - dont j'ai déjà indiqué la prégnance - peut d'ailleurs fort bien être considérée sous cet angle, autant éthique qu'esthétique."

19. Idem, p.202.

20. Cf., idem, p.157: "L'invention du document et la fiction de l'archive manifestent bien l'ambition du roman contemporain face à l'histoire: par elles, le roman entend retrouver le texte perdu de l'histoire, pour le produire sur la scène publique"

21. Cf,.Maria de Fátima Marinho, op.cit. e Um Poço sem Fundo - Novas Reflexões sobre Literatura e História, Porto, Campo das Letras, 2005.

22. António Lobo Antunes, As Naus, Lisbonne, Publicações Dom Quixote, 1988, p.200.

23. Luís de Camões.

24. Lieu où est apparu un faux roi Sebastião, après la débâcle d'Alcácer Quibir.

25. As Naus, p.240.

26. António Lobo Antunes, O Esplendor de Portugal, Lisbonne, Publicações Dom Quixote, 1997, p.51.

27. Idem, p.79.

28. Idem, p.121.

29. Idem, p.356.

30. Idem, p.387.

31. Lídia Jorge, O Vento Assobiando nas Gruas, Lisbonne, Dom Quixote, 2002, p.15

32. Idem, p.161.

33. Idem, p.529.

34. Nuno Júdice, Por Todos os Séculos, Lisbonne, Quetzal Editores, 1999, p.13.

35. Agustina Bessa-Luís, A Ronda da Noite, Lisboa, Guimarães Editores, 2006, p.15.

36. Idem, p.199.

37. Idem, p.119. 
38. Idem, p.301.

39. Idem, p.359.

\section{RÉSUMÉS}

Dans ce petit essai, on étudiera le rôle des grands récits dans l'établissement d'une identité qui risque de se dissoudre après les transformations politiques et sociales du début du XIX ${ }^{\mathrm{e}}$ siècle. Après une analyse rapide des modes de production romantiques et des premières années du siècle dernier, on arrive aux romans contemporains et à la problématisation de l'identité qui en découle. On étudie trois romans (As Naus et $O$ Esplendor de Portugal, de Lobo Antunes et $O$ Vento Assobiando nas Gruas, de Lídia Jorge) qui parlent du rapport entre les portugais et leurs anciennes colonies africaines. La vraie compréhension du passé ne se complète qu'avec le mélange des temps et l'indistinction des personnages appartenant à des époques différentes. C'est le cas de plusieurs romans d'Agustina Bessa-Luís (O Mosteiro (1980), o Concerto dos Flamengos (1995) ou Ordens Menores (1992)), qui reflètent le devenir historique. L'analyse des grands récits se termine par l'approche de A Ronda da Noite, dernière oeuvre d'Agustina. Dans cette oeuvre, on essayera d'établir les modes qui déterminent la recherche de l'identité à travers le reflet fragmentaire d'une réalité fuyante. Les exemples cités démontrent le besoin d'atteindre le secret du moi et de l'autre, secret fondamental pour l'établissement d'une identité toujours menacée.

\section{AUTEUR}

\section{MARIA DE FÁTIMA MARINHO}

Université de Porto 